J.Prause, Y. Emmi, B. Noll, M. Aigner, LES/RANS Modeling of Turbulent Mixing in a Jet in Crossflow at Low Velocity Ratios, In: Proceedings. 54th AIAA Aerospace Sciences Meeting, 04.-08.01.2016, San Diego (USA).

The AIAA version of the paper is accessible at http://dx.doi.org/10.2514/6.2016-0609

On the AIAA web page http://www.aiaa.org/content.cfm?pageid=2

the interested reader can find other material published by AIAA 


\title{
LES/RANS Modeling of Turbulent Mixing in a Jet in Crossflow at Low Velocity Ratios
}

\author{
Juliane Prause*, Yeshaswini Emmi*, Berthold Noll ${ }^{\dagger}$ and Manfred Aigner ${ }^{\ddagger}$ \\ German Aerospace Center (DLR), Stuttgart, Germany
}

\begin{abstract}
Turbulent mixing of jets in crossflow plays a crucial role in many technical applications, such as fuel injection in internal combustion systems, film cooling in gas turbines or environmental pollution discharge from piping systems. In the present investigation, numerical simulations at jet to crossflow velocity ratios of 0.5 and 2 were conducted at Reynolds numbers of 20500 and 82000 respectively. Three different turbulence models are applied and compared with respect to model capabilities and costs. The main focus is on the validation of the Scale-Adaptive Simulation (SAS) model. It is a hybrid of the Reynolds-averaged Navier-Stokes (RANS) method and the Large Eddy Simulation (LES). The capabilities are compared with pure the RANS and LES modeling. Special attention is payed to the modeling accuracy in the upper shear layer, recirculation zone and wake. The numerical results are compared with experimental data for the velocity and mixture fields. For both velocity ratios, the SAS stays in RANS mode in the upper shear layer, as well as in the recirculation zone for velocity ratio 0.5 . In contrast, at the higher velocity ratio, the SAS model reproduces the recirculation zone with LES quality. For both velocity ratios, the accuracy achieved in the wake by the SAS model is comparable with LES.
\end{abstract}

\section{Nomenclature}

Symbols

$D \quad$ jet pipe diameter, $\mathrm{m}$

jet jet pipe Stream

$k$ turbulent kinetic energy, $\mathrm{m}^{2} / \mathrm{s}^{2} \quad$ res resolved

mod modeled

$Q \quad$ 2nd invariant of vel. gradient tensor, $1 / s^{2}$

$R \quad$ velocity ratio

Re Reynolds number

$S \quad$ strain rate, $1 / \mathrm{s}$

$T$ temperature, $\mathrm{K}$

$t \quad$ turbulent

$x, y, z \quad$ stream-, length-, spanwise coordinates, $\mathrm{m} / \mathrm{s}$

$u, v, w \quad$ stream-, length-, spanwise velocities, $\mathrm{m} / \mathrm{s}$

$Z \quad$ mixture fraction

Operators

() $\quad$ filtered variable

() $\quad$ Favre-averaged variable

$\mu \quad$ dynamic viscosity, $\mathrm{kg} / \mathrm{m} \mathrm{s}$

$\omega \quad$ specific dissipation rate, $1 / \mathrm{s}$

Abbreviations

$C F D \quad$ Computational Fluid Dynamics

LES Large Eddy Simulation

$S A S \quad$ Scale-Adaptive Simulation

SST Shear Stress Transport

Subscript

cf crossflow

RANS Reynolds-Averaged Navier-Stokes

$W A L E$ Wall-Adapting Local Eddy-Viscosity

\section{Introduction}

The present investigation is part of a research project ${ }^{1}$ on fuel injection in the Alstom Reheat Gas Turbine GT24/26. ${ }^{2}$ In this project hydrogen fuel is injected perpendicular to a hot oxidizer stream. Fast

\footnotetext{
${ }^{*}$ Research Scientist, Institute of Combustion Technology, Pfaffenwaldring 38-40, 70569 Stuttgart, Germany.

${ }^{\dagger}$ Head of Department Numerical Simulation, Institute of Combustion Technology, Pfaffenwaldring 38-40, 70569 Stuttgart, Germany.

${ }^{\ddagger}$ Head of Institute, Institute of Combustion Technology, Pfaffenwaldring 38-40, 70569 Stuttgart, Germany.
} 
chemical reactions take place in the mixing region, which can induce premature autoignition events in the mixing duct upstream of the combustion chamber. Accurate prediction of turbulent mixing is crucial to avoid autoignition in the mixing duct. To this end, the model capabilities in the upper shear layer are of special interest, since autoignition favors very lean conditions (mixture fraction $Z<0.1$ ). Additionally, the recirculation zone is very critical due to possible flame stabilization processes, as shown by Fleck et al. ${ }^{3}$

The technical system is characterized by high pressures and temperatures as well as high Reynolds numbers. Furthermore, the mixing process is superimposed by wall cooling and chemical reaction. Due to these challenging conditions, detailed measurement of the velocity and mixing field is not possible. Beyond that, information about the boundary and inflow conditions is limited. ${ }^{1}$ Due to the high uncertainty in the reference data, an accurate validation of the turbulence models is not possible at real technical conditions. Therefore a generic test case has been selected to gain a better understanding of the turbulence model capabilities. Characteristic flow quantities are maintained between the generic test setup and the combustion chamber application case, such as the momentum ratio and level of turbulence. However, in the selected experiment by Andreopoulos and Rodi, ${ }^{4,5}$ pressure and temperature are atmospheric and the gas consists of pure air. Due to these simplified conditions, detailed and reliable validation data of the velocity and mixing field could be obtained. The reference data comprises averages, higher moments and scalar fluxes at high Reynolds numbers and with well defined boundary conditions. It has been demonstrated in several previous studies $^{6-8}$ that this test case can be reproduced well with LES.

For modeling turbulent flows in industrial applications, RANS models are still the prevailing method. ${ }^{9}$ Although this method is very reliable for one-dimensional flows, RANS-based models have deficiencies in capturing three-dimensional flow structures (e.g. separation zones) due to the underlying eddy viscosity approach. These flow types can be better reproduced by LES approaches, where large turbulent structures are directly resolved. However, a high grid resolution is required and LES can become prohibitively expensive for technical applications with high turbulent Reynolds numbers. A very convenient alternative is offered by hybrid RANS/LES modeling. One-dimensional or less important flow regions are modeled by cost-efficient RANS methods, while the scale-resolving (LES-like) mode is applied for relevant three-dimensional flow regions. In the present investigation the SAS approach by Menter and Egorov ${ }^{10}$ is applied to model the transition between RANS and LES interfaces. This model has the advantage that the RANS mode switches to scale-resolving mode automatically as soon as large unsteady turbulent structures can be resolved by the mesh; in addition, no rigid predefined interface is required. It was shown by Ivanova ${ }^{11}$ that SAS modeling is more accurate compared to RANS modeling for transverse jets at intermediate velocity ratios. However, the automatic switch from steady-state to scale-resolving mode is initiated by large unsteady structures. The transition at the interface is therefore not well defined, especially for weak flow instabilities as present in the shear layer at low momentum jets as has been shown by several studies. ${ }^{12-15}$ Though, these investigations were mainly based on applied configurations. The present study aims to analyze the capabilities of SAS, URANS and LES turbulence models in a generic test case. Of special interest are the capabilities in the upper shear layer and the recirculation zone and the potential influence on chemical reaction.

\section{Reference Data}

The generic turbulent jet in crossflow configuration by Andreopoulos and Rodi ${ }^{4,5}$ is applied as reference for model validation. The experiment was conducted in a closed-circuit wind tunnel with air at atmospheric conditions. Since the density is almost identical for both streams, the momentum ratio corresponds to the velocity ratio $R$. The Reynolds number is $R e=82000$ for a velocity ratio of $R=2$, based on a pipe diameter of $D=50 \mathrm{~mm}$ and a mean jet velocity of $27.8 \mathrm{~m} / \mathrm{s}$. The Reynolds number for $R=0.5$ is $R e=20500$. The jet stream is heated $4 K$ above the ambient crossflow temperature. The steady mixture field is then assessed by thermocouple measurements. Temperature fluctuations were measured by cold-wire probes, the velocity

fields were determined by cross-wire probes. The boundary conditions are adequately defined. Both streams are developing flows. The pipe flow has a length of $12 D$ from plenum to jet exit. The crossflow is defined by a boundary layer thickness of $0.278 D$ at $4 D$ upstream of the jet exit.

\section{Modeling Approach}

All computations are performed with the DLR CFD code THETA. 


\section{Computational Set-Up}

The computational domain has following dimensions: streamwise $-2<x / D<7$, lengthwise $-2<y / D<$ 24.4, spanwise $-6<z / D<6$ with its point of origin in the center of the pipe exit plane. The grid resolution of the main flow region is consistent for the computations of all three turbulence models. In the jet mixing region $(-2<x / D<7, y / D<4$ and $-2<z / D<2)$, the unstructured mesh has a grid size of $1.5 \mathrm{~mm}$. However, different resolutions are applied adjacent to the walls. For the LES computation the turbulent boundary layer at the walls is fully resolved. Here the first wall cell height is set to $y_{1}^{+} \approx 2$. This results in a total number of about 30 million grid points. The advantage of SAS and (U)RANS models is a the possible reduction of computational cells in one-dimensional flow regions. A large number of cells can be saved, if the turbulent wall layer is modeled by wall functions. With this approach the first wall cell height can be set to $y_{1}^{+} \approx 30$. With a resolution of $1.5 \mathrm{~mm}$ in the mixing region $\left(y^{+} \approx 60\right.$ with respect to crossflow), the final grid for SAS and (U)RANS consists of about 10 million grid points. Further reduction of the cell number could be achieved by grid coarsening upstream of the jet-crossflow interface. To obtain the velocity fields at the inlet additional LES were conducted for a flat plate and a turbulent pipe inflow. Time-resolved slices are extracted and applied to the inlet boundaries in accordance to the experimental measurements. Unsteady fields with resolved fluctuations are applied for LES while for RANS and SAS the LES inflow data is used to generate steady profiles of averaged velocities and turbulent quantities.

\section{Turbulence Models}

\section{RANS-SST}

As described above different turbulent models are applied. As can be seen in the results section, knowledge of the statistical flow properties is sufficient for many applications. Therefore the Navier-Stokes equations can be averaged, called RANS modeling. In the averaged equation an unclosed Reynolds stress tensor $\tau_{i j}$ occurs, which can be modeled by the eddy viscosity approach.

$$
\tau_{i j}=\bar{\rho}\left(\overline{u_{i}^{\prime \prime} u_{j}^{\prime \prime}}\right) \approx \mu_{t}\left(\frac{\partial \overline{u_{i}}}{\partial x_{j}}+\frac{\partial \overline{u_{j}}}{\partial x_{i}}\right)
$$

In the present work the the two-equation Shear Stress Transport (SST) model by Menter ${ }^{16}$ is applied for closure of the turbulent viscosity $\mu_{t}$. This model combines the advantages of the Wilcox $k-\omega$ turbulence model $^{17}$ in proximity to walls with the standard $k$ - $\epsilon$ model $^{18}$ in the mean flow field by introducing an automatic blending between both models ( $F 1$ and $F 2$ ).

$$
\mu_{t}=\min \left(\rho \frac{k}{\omega}, \rho \frac{a_{1} k}{F_{2} S}\right)
$$

With strain rate $S=\sqrt{2 S_{i j} S_{i j}} \quad$ and $\quad S_{i j}=\frac{1}{2}\left(\frac{\partial u_{i}}{\partial x_{j}}+\frac{\partial u_{j}}{\partial x_{i}}\right)$.

The two transport equation for $k$ and $\omega$ are derived from from the Navier-Stokes equations.

$$
\begin{gathered}
\frac{\partial(\rho k)}{\partial t}+u_{j} \frac{\partial(\rho k)}{\partial x_{j}}-\frac{\partial}{\partial x_{j}}\left[\left(\mu+\sigma_{k} \mu_{t}\right) \frac{\partial k}{\partial x_{j}}\right]=\tilde{P}_{k}-\beta^{*} \rho k \omega \\
\frac{\partial(\rho \omega)}{\partial t}+u_{j} \frac{\partial(\rho \omega)}{\partial x_{j}}-\frac{\partial}{\partial x_{j}}\left[\left(\mu+\sigma_{\omega} \mu_{t}\right) \frac{\partial \omega}{\partial x_{j}}\right]=\gamma \frac{\rho}{\mu_{t}} \tilde{P}_{k}-\beta \rho \omega^{2}+2\left(1-F_{1}\right) \rho \sigma_{\omega_{2}} \frac{1}{\omega} \frac{\partial k}{\partial x_{j}} \frac{\partial \omega}{\partial x_{j}}
\end{gathered}
$$

The exact description of the blending function $F 1$ and $F 2$, the limited production term $\tilde{P}_{k}$ as well as the model constants $a_{1}, \sigma_{k}, \sigma_{\omega}, \sigma_{\omega_{2}}, \beta$ and $\beta^{*}$ can be found in the publication by Menter et al. ${ }^{19}$ The RANS computations are performed time-resolved to ensure a good comparability with the likewise unsteady SAS runs. Since the validity of unsteady (U)RANS is questioned by some authors, ${ }^{9}$ additional steady RANS computations are also performed. 


\section{SAS-SST}

The SAS model is an extension of the SST model.

$$
P_{S A S}=\max \left[\hat{\zeta} \kappa S \frac{L_{t}}{L_{v k}}-C \frac{2}{\sigma_{\Phi}} k \max \left(\frac{1}{\omega^{2}} \frac{\partial \omega}{\partial x_{j}} \frac{\partial \omega}{\partial x_{j}}, \frac{1}{k^{2}} \frac{\partial k}{\partial x_{j}} \frac{\partial k}{\partial x_{j}}\right), 0\right]
$$

model constants

$$
\hat{\zeta}=3.52, C=2, \kappa=0.41, \sigma_{\Phi}=2 / 3
$$

von Karman length scale $L_{v K}$

$$
L_{v K}=\max \left(\kappa \frac{S}{\sqrt{\frac{\partial^{2} \overline{u_{i}}}{\partial x_{k}^{2}} \frac{\partial^{2} \overline{u_{i}}}{\partial x_{j}^{2}}}}, C_{s} \sqrt{\frac{\kappa \hat{\zeta}}{\beta_{\omega 2} / \beta_{k}-\gamma_{2}} \Delta}\right)
$$

The constants $\beta_{w 2}, \beta_{k}$ and $\gamma_{2}$ are set according to the SST-model. $C_{s}$ is set to 0.145 .

An additional production term $P_{S A S}$ is added to the right-hand side of the w-equation 4 . This term depends on the second velocity derivate and therefore becomes effective when large unsteady structures can be resolved by the grid. The model then switches into scale-resolving mode where it is able to provide a broad turbulent spectrum, similar to LES. This extension allows to apply RANS methods in steady flows and close to walls. In large unstable separation regions the model automatically switches into scale-resolving mode, if the grid is resolved sufficiently. The model is implemented as proposed by Menter and Egorov. ${ }^{10}$

\section{LES-WALE}

The most sophisticated turbulence model applied in the present contribution is the LES method. In LES a spatial filter function is applied to the Navier-Stokes equations. The filter width is usually similar to the size of the grid cells. The full turbulent spectrum of turbulent scales is directly resolved in the whole domain up to the length of the grid cells. The impact of the unresolved scales smaller than the is modeled by a subgrid model. The filtered Navier-Stokes equations are very similar in structure to the RANS equations. In the present contribution the unclosed subgrid scale stress tensor $\tau_{i j, s g s}$ is modeled by the Wall-Adapting Local Eddy-Viscosity (WALE) model by Ducros et al. ${ }^{20}$

$$
\begin{gathered}
\tau_{i j, s g s}=\mu_{t}\left(\frac{\partial \widetilde{u}_{i}}{\partial x_{j}}+\frac{\partial \widetilde{u}_{j}}{\partial x_{i}}\right) \\
\mu_{t}=\bar{\rho}\left(\Delta C_{w}\right)^{2} \frac{\left(S_{i j}^{d} S_{i j}^{d}\right)^{3 / 2}}{\left(\widetilde{S}_{i j} \widetilde{S}_{i j}\right)^{5 / 2}+\left(S_{i j}^{d} S_{i j}^{d}\right)^{5 / 4}} \\
S_{i j}^{d}=\frac{1}{2}\left(\frac{\partial \widetilde{u}_{i}}{\partial x_{k}} \frac{\partial \widetilde{u}_{k}}{\partial x_{j}}+\frac{\partial \widetilde{u}_{j}}{\partial x_{k}} \frac{\partial \widetilde{u}_{k}}{\partial x_{i}}\right)-\frac{1}{3} \delta_{i j}\left(\frac{\partial \widetilde{u}_{k}}{\partial x_{k}}\right)^{2}
\end{gathered}
$$

The WALE coefficient is set to 0.325 .

\section{Scalar Transport}

The scalar heat transport is described by an additional transport equation for the temperature. In the statistical equation the turbulent heat fluxes $q_{i, t}$ must be closed. Similar to the Boussinesq hypothesis it is assumed that the flux is proportional to the temperature gradient. The turbulent thermal diffusivity $a_{t}$ is modeled by the Reynolds analogy with a turbulent Prandtl number $P r_{t}=1.0$.

$$
q_{i, t}=a_{t} \frac{\partial \bar{T}}{\partial x_{i}}=\frac{\mu_{t} / \rho}{P r_{t}} \frac{\partial \bar{T}}{\partial x_{i}}
$$




\section{Results}

\section{Velocity Ratio $R=2.0$}

The time-averaged distributions for mixture fraction $Z$ and dimensionless streamwise velocity $u / u_{c f}$ are presented in figures 1 and 2 . The section is located at the plane of symmetry $z / D=0$. The dimensionless mixture fraction $Z$ is defined by equation 12. It ranges from 0 to 1 and is, by definition, 0 in the pure crossflow and 1 in the jet pipe.

$$
Z=\frac{T-T_{c f}}{T_{j e t}-T_{c f}}
$$
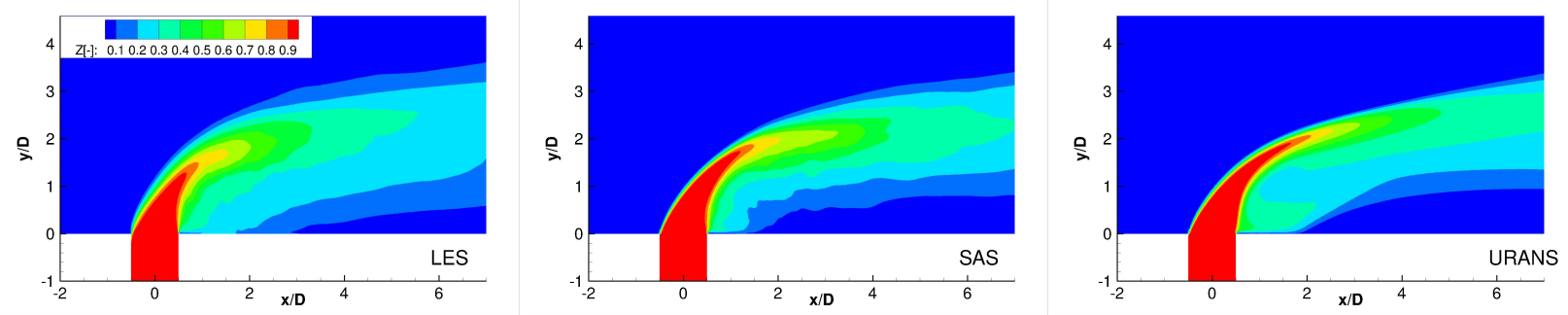

Figure 1. $R=2$ : Time-averaged mixture fraction $Z$ at plane of symmetry $z / D=0$.
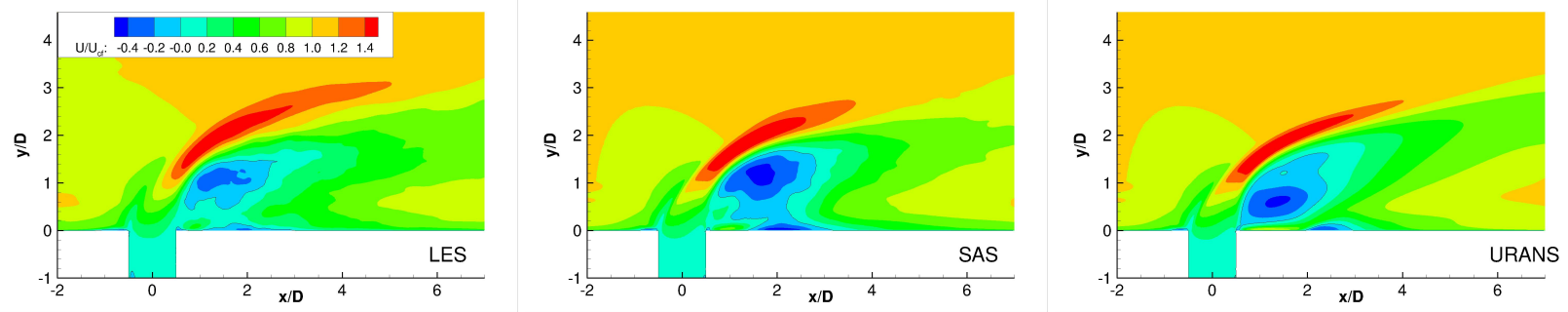

Figure 2. $R=2$, Time-averaged dimensionless streamwise velocity $\bar{u} / u_{c f}$ at plane of symmetry $z / D=0$.

At this relatively low velocity ratio, the jet is bent strongly by the crossflow. The jet in turn displaces the crossflow. This leads to increased streamwise velocities in the upper shear layer. A large recirculation zone forms on the downwind side of the jet and a second small recirculation zone can be found close to the wall. Downstream, the wake is lifted from the wall and crossflow fluid pours inwards below the wake.
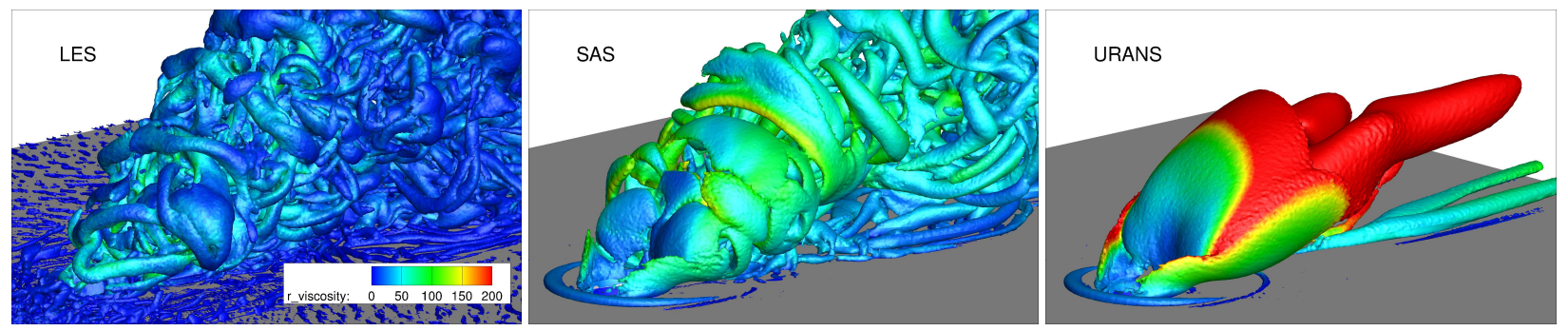

Figure 3. $R=2$ : Isosurface of $Q$-criterion at $Q=10000$ colored with viscosity ratio.

First, the modeling of the turbulent mixing is investigated in the upper shear layer. The instantaneous examples in figures 3 and 4 give an impression of the turbulent structures for all three turbulence models. In figure 3 vortices are visualized by the $Q$-criterion ${ }^{21}$ at a level of $Q=10000$. The isosurface is colored with the viscosity ratio. It is an indicator for the amount of resolved turbulence and is defined as the quotient of turbulent and molecular viscosity. The smaller the ratio, the more turbulence is resolved. The corresponding instantaneous mixture fraction distributions are presented in figure 4. With LES (left) a broad range of turbulent structures is resolved. Also the roll-up of jet shear layer vortices is captured in the windward jet side upstream of $x / D=1$ (cp. figure 4). The SAS model resolves only very large structures 

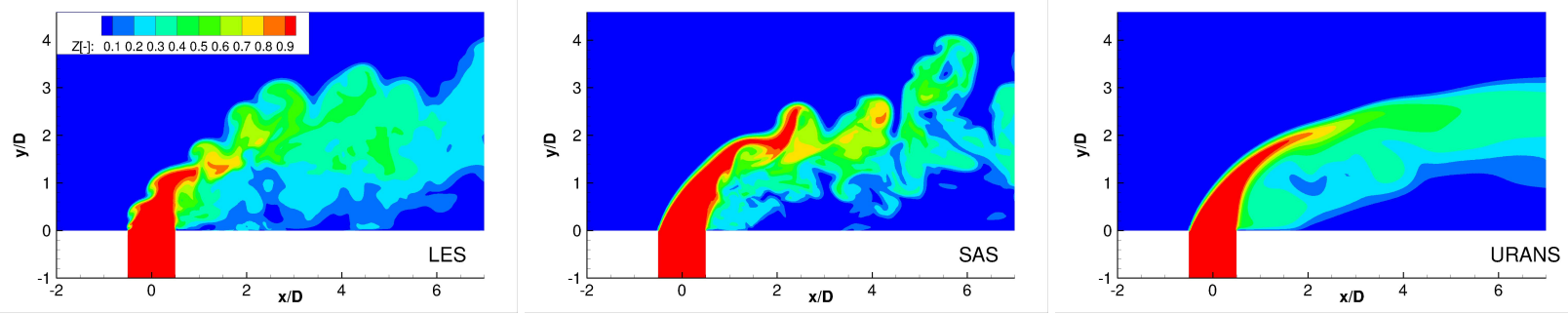

Figure 4. $R=2$ : Instantaneous mixture fraction $Z$ at plane of symmetry $z / D=0$.

in the upper mixing region. Especially the upper shear layer does not start to roll up before $x / D=1$. Downstream also smaller vortical structures are resolved. Finally, the results with the time-resolved RANS model are presented on the right. Here only the largest structures are reproduced, such as the horseshoe and the counter rotating vortices. ${ }^{22}$ A spectrum of turbulent scales is not captured at all.
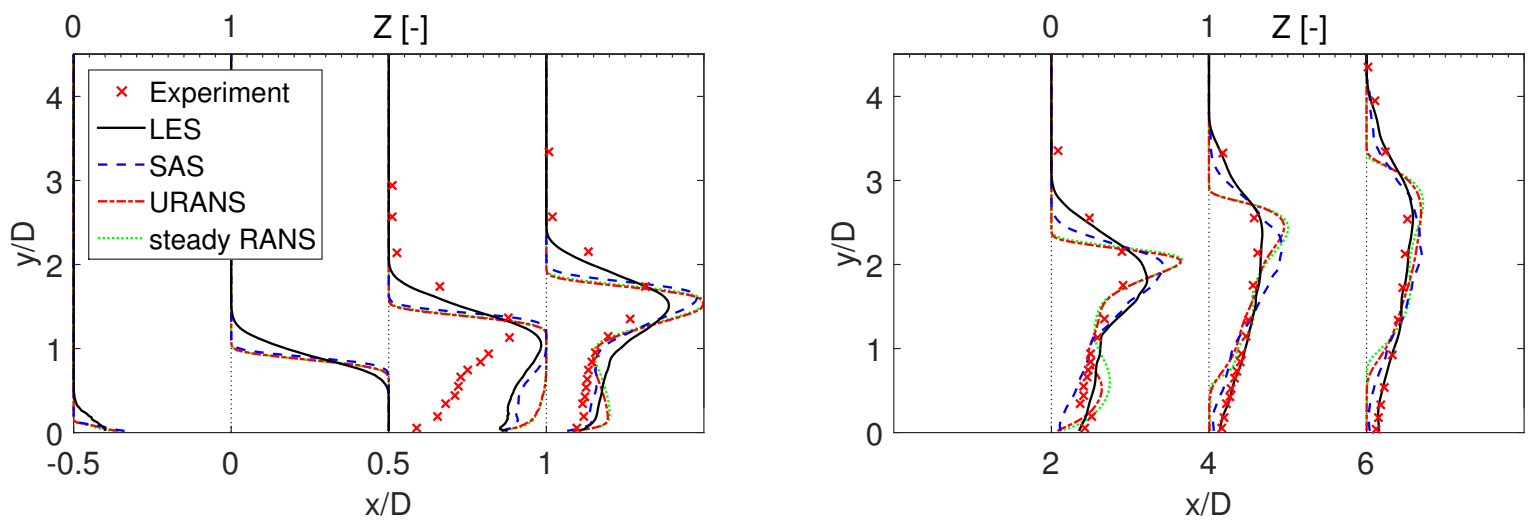

Figure 5. $R=2$ : Profiles of time-averaged mixture fraction $Z$ at plane of symmetry $z / D=0$ and different streamwise locations $x / D=-0.5$ to 1 (left) and $x / D=2$ to 6 (right).
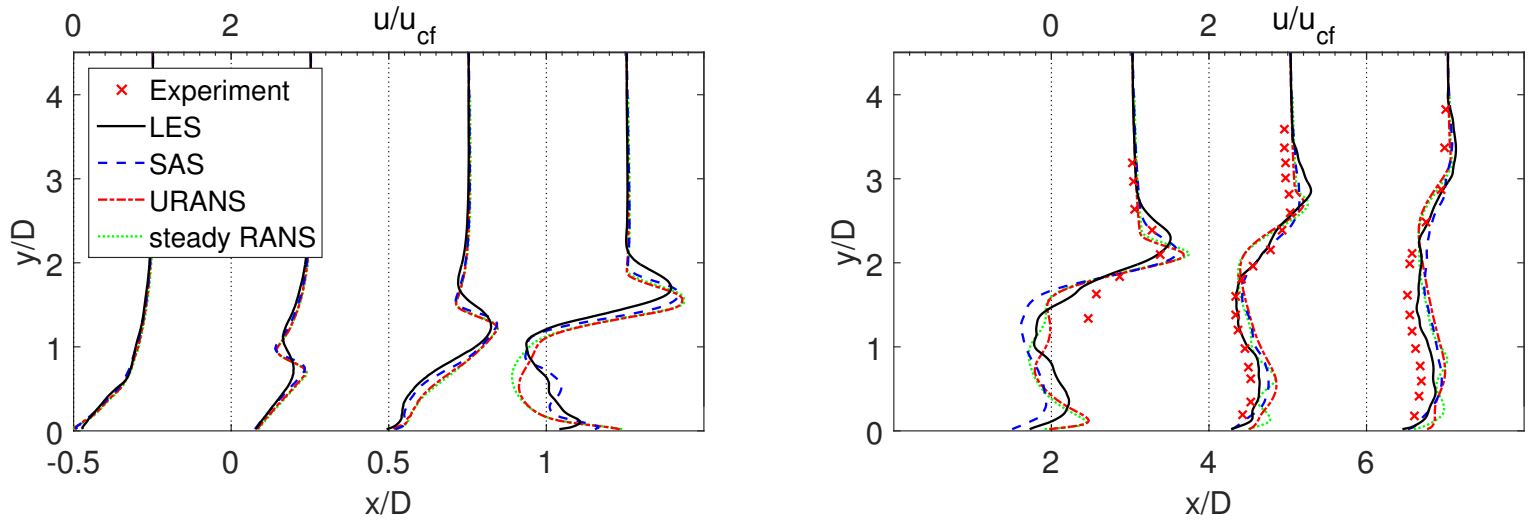

Figure 6. $R=2$ : Profiles of time-averaged dimensionless streamwise velocity $\bar{u} / u_{c f}$ at plane of symmetry $z / D=0$ and different streamwise locations $x / D=-0.5$ to 1 (left) and $x / D=2$ to 6 (right).

The time-averaged mixture fraction and velocity profiles are shown in figures 5 to 8 . The profiles are plotted at 7 streamwise locations at the plane of symmetry $z / D=0$. The streamwise position is given at the bottom x-axis, the scaling of the parameters can be found at the top. The LES results agree well with the experimental data in the upper shear layer and are in the range of experimental uncertainties. The URANS and steady RANS results are very similar. The gradients of mixture fraction and velocities are very high. The eddy-viscosity approach does not capture the mixing sufficiently. The model works well if the flow is one-dimensional, such as in turbulent boundary layers. However, large three-dimensional vortices are present in the jet shear layer (compare figures 1 and 2 left). Since these are not resolved, the turbulent fluctuations 

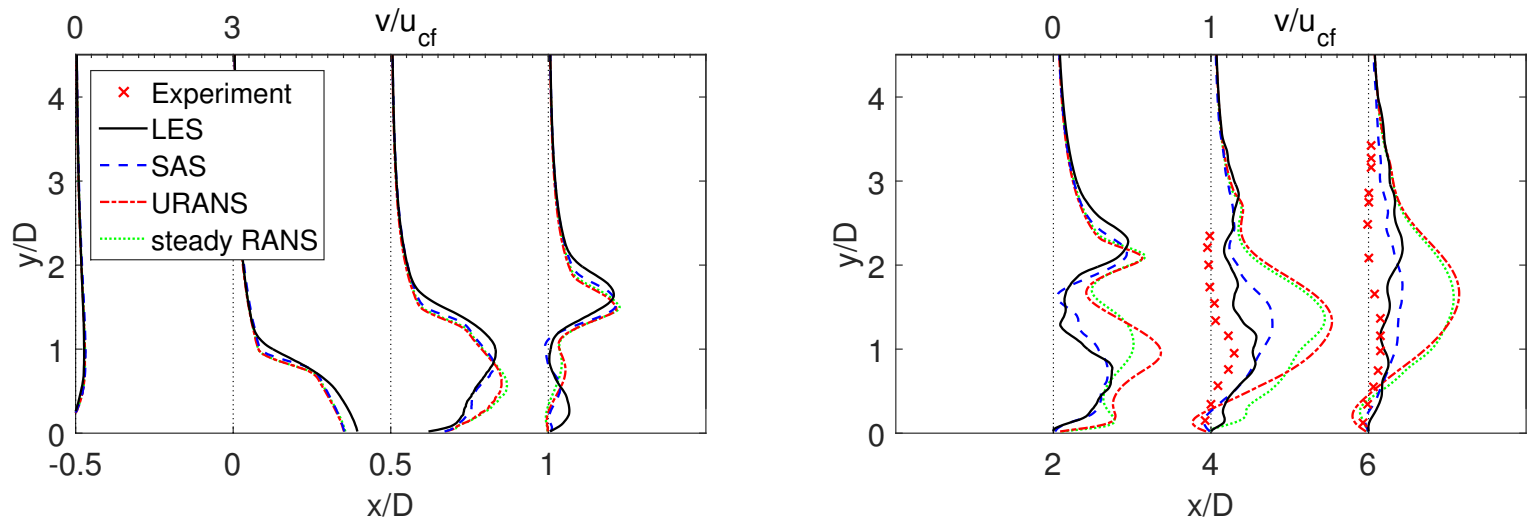

Figure 7. $R=2$ : Profiles of time-averaged dimensionless streamwise velocity $\bar{v} / u_{c f}$ at plane of symmetry $z / D=0$ and different streamwise locations $x / D=-0.5$ to 1 (left) and $x / D=2$ to 6 (right).
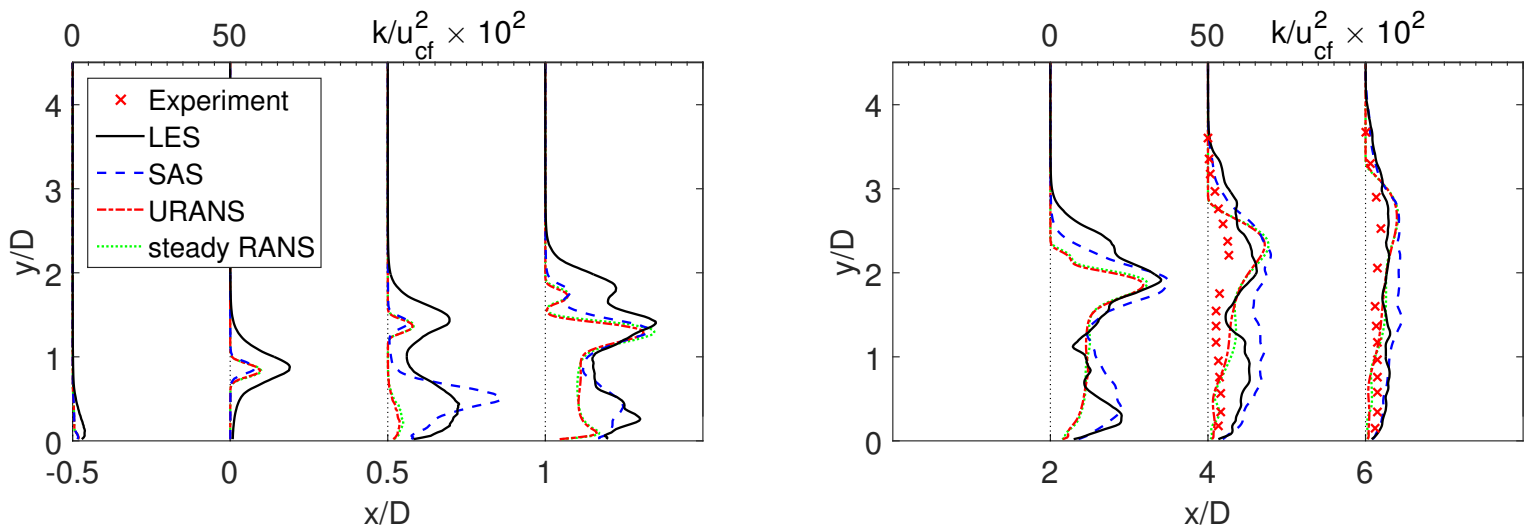

Figure 8. $R=2$ : Profiles of time-averaged dimensionless turbulent kinetic energy $k / u_{c f}^{2}$ at plane of symmetry $z / D=0$ and different streamwise locations $x / D=-0.5$ to 1 (left) and $x / D=2$ to 6 (right).

are underpredicted in the shear layer as shown in figure 8. Therefore turbulent mixing is underestimated by the model. Also further downstream in the lifted wake, the upward motion is overpredicted by the RANS approach. The SAS model is in RANS-mode at the front of the upper shear layer. At about $x / D=2$ it starts to switch to scale-resolving mode and more and more vortices are resolved. In the wake the mixture fraction distribution is similar to LES.

Good agreement with experimental data is achieved for the mixture fraction in the recirculation zone $(x / D<2, y / D<3)$ for all three turbulence models (cp. figure 5$)$. The temperature is slightly overpredicted at the right wall of the jet exit $x / D=0.5$. This can be attributed to potential wall cooling in the experiment, which is not considered in the simulation or uncertainties in the pipe inflow conditions. Unfortunately, no experimental data was collected for velocity quantities in the recirculation zone. Nevertheless, the SAS results are similar to the LES results for velocities and mixture fraction downstream of the jet exit at $x / D=0.5$. In contrast, the RANS results differ from LES and SAS results in the three-dimensional recirculation zone. In the two-dimensional velocity plots in figure 2 the locations of the two recirculation zones are on top of each other and at the same streamwise location with SAS and LES. With RANS the large recirculation zone is located more upstream and closer to wall and the lower recirculation zone is much smaller. The region around $x / D=2$ close to the wall is of special interest for potential flame stabilization processes in premixed combustion systems ${ }^{3}$ as discussed below. In figure 6 , it is evident that the velocities are negative at this location for SAS and LES, whereas the streamwise velocity is positive for RANS. Again, there is almost no difference between the steady RANS and URANS results. 
Velocity Ratio $R=0.5$

At $R=0.5$ the jet velocity is two times smaller than the cross stream. The jet is bent even more by the crossflow than in the previous case. The recirculation zone is smaller (cp. figure 10) and the mixing process takes place mainly very close to the lower wall. Downstream of the recirculation zone $(x / D \leq 2)$ the wake is even pressed downwards (cp. figure 10, LES).
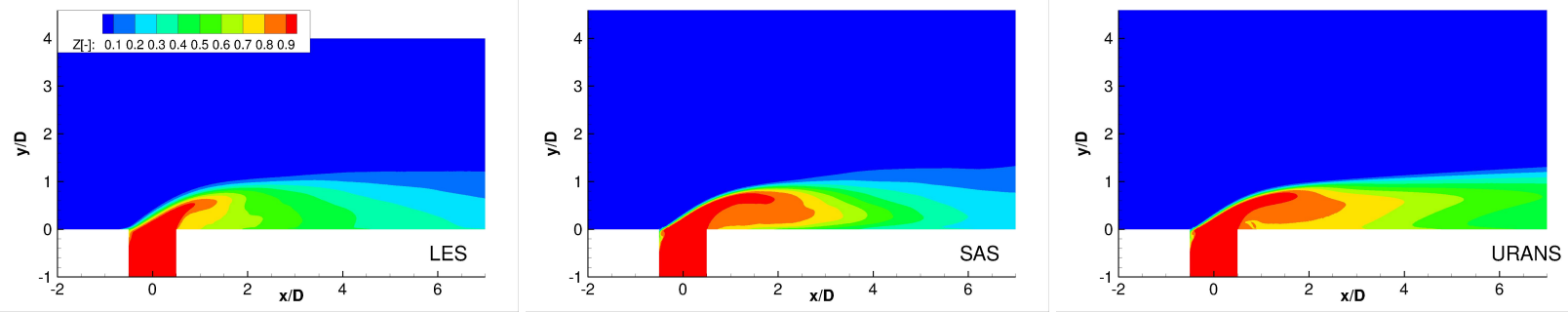

Figure 9. $R=0.5$ : Time-averaged mixture fraction $Z$ at plane of symmetry $z / D=0$.
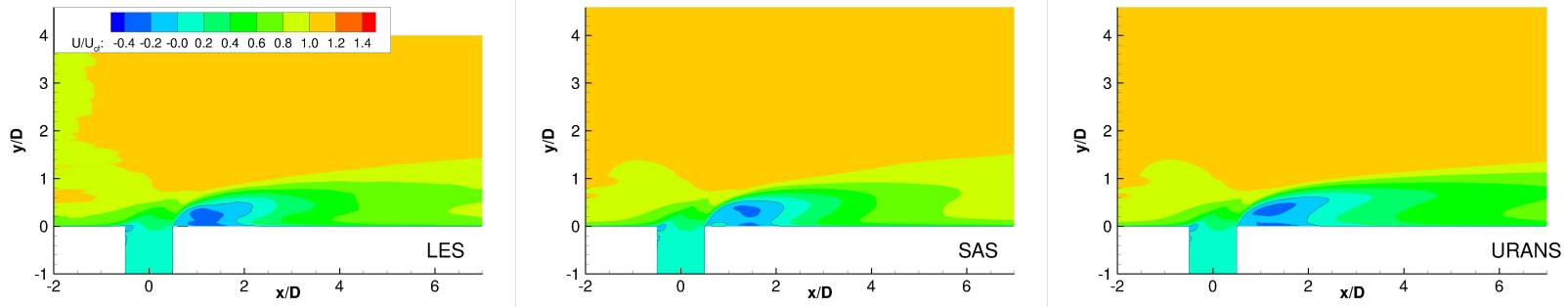

Figure 10. $R=0.5$ : Time-averaged dimensionless streamwise velocity $\bar{u} / u_{c f}$ at plane of symmetry $z / D=0$.
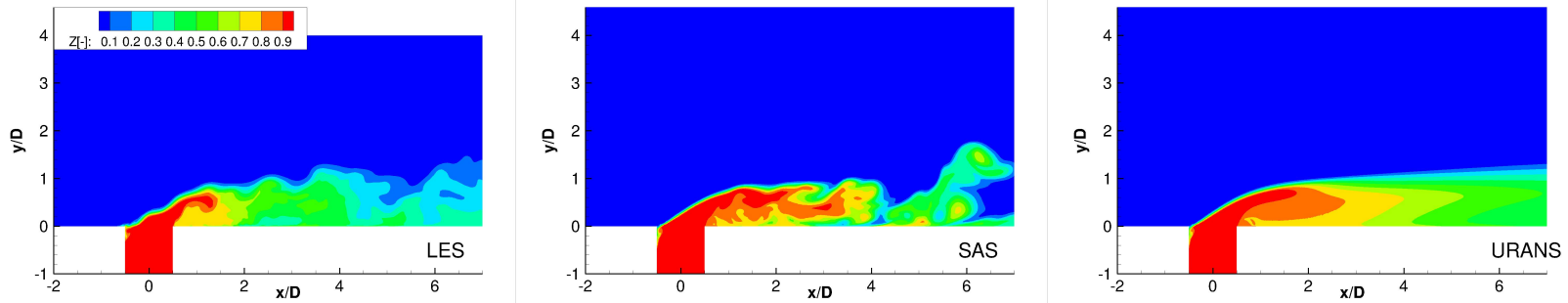

Figure 11. $R=0.5$ : Instantaneous mixture fraction $Z$ at plane of symmetry $z / D=0$.

Although the overall form is slightly different to the $R=2$ case, similar model characteristics are evident from the instantaneous mixture fraction plots in figure 11. With LES, turbulent structures are captured at the leading jet-crossflow interface, although the roll-up of the shear layer is less pronounced. With SAS the front zone of the upper shear layer is still quite smooth and no distinct vortices are resolved. After a short distance, the SAS switches to the scale-resolving mode. However, the instantaneous mixing field seems to be less stirred compared to LES. And again, almost no time-dependent vortices are resolved with the RANS model, not even in the unsteady simulation.

A detailed comparison with experimental data is shown in figures 12 to 15 . The mixing and velocity fields in the upper shear layer are very well reproduced with LES. The average velocity profiles (in figure 13) are very similar for all three turbulence models in the upstream part of the jet up to $x / D=1$. This is very similar to the $R=2$ case. In contrast, turbulent mixing (figure 13) is underpredicted with RANS and SAS in the upper shear layer due to underprediction of turbulent kinetic energy. In the wake, downstream of $x / D=2$, the SAS results approach the LES and experimental data and a good agreement is achieved at $x / D=6$. At this location the mixing process is advanced quite far and the gradients of mixture fraction and velocity are small. However, the RANS model does not reproduce this feature.

In the recirculation zone $(x / D=1-2$ and $y / D<0.5)$, high accuracy is achieved for the mixture fraction (cp. figure 12). The difference in temperature at $x / D=0.5$ have also been observed in the $R=2$ 

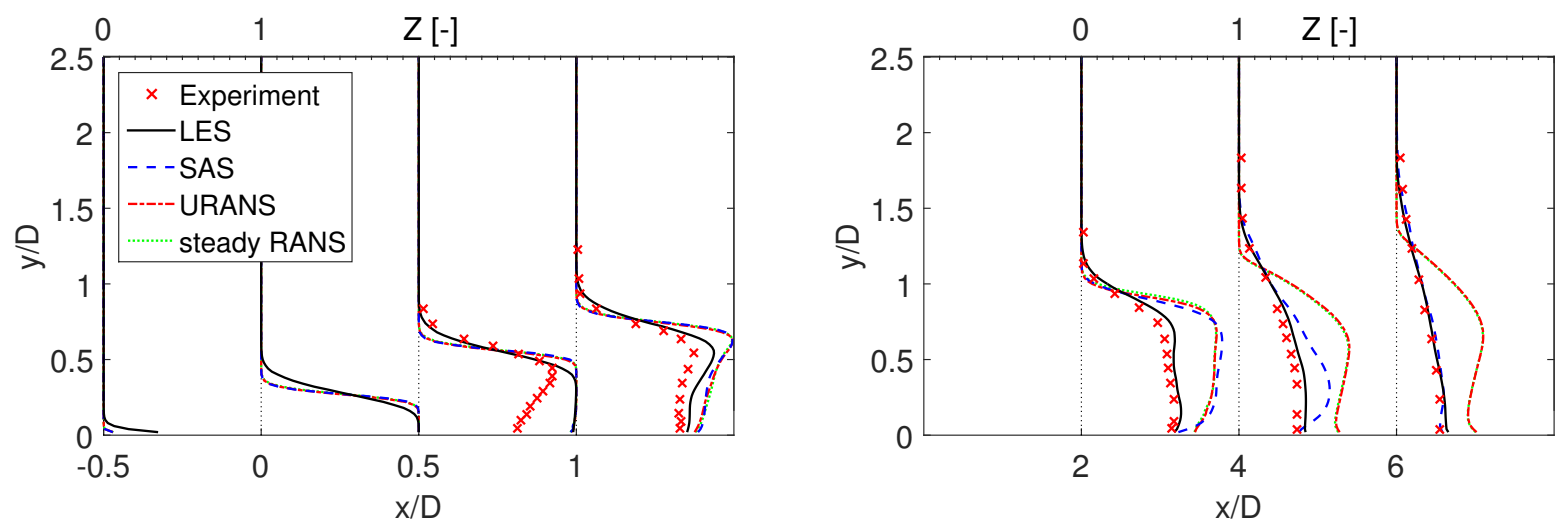

Figure 12. $R=0.5$ : Profiles of time-averaged mixture fraction $Z$ at plane of symmetry $z / D=0$ and different streamwise locations $x / D=-0.5$ to 1 (left) and $x / D=2$ to 6 (right).
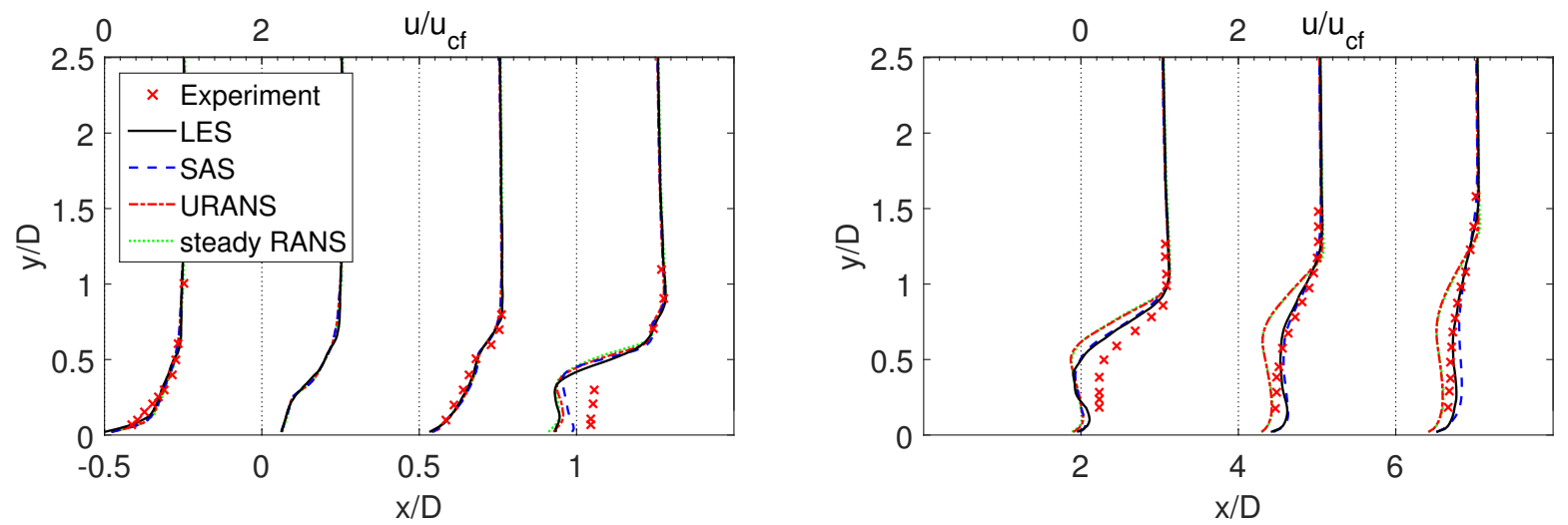

Figure 13. $R=0.5$ : Profiles of time-averaged dimensionless streamwise velocity $\bar{u} / u_{c f}$ at plane of symmetry $z / D=0$ and different streamwise locations $x / D=-0.5$ to 1 (left) and $x / D=2$ to 6 (right).

case. It might be due to heat transfer at the wall as described above. There are larger deviations for the streamwise velocities at $x / D=1$. In the experiment the streamwise velocity $u$ has a different sign. However, the magnitude corresponds quite well. Furthermore, it seems that the turbulent kinetic energy is too high in the LES and SAS simulations. Similar results were obtained by Schlüter and Schönfeld ${ }^{6}$ as well as by Wegner et. al. ${ }^{7}$ As already explained by these authors, the hot-wire measurement which is applied in the experiment does not measure flow directions. Furthermore, Andreopoulos ${ }^{4}$ mentions that the hotwire measurement underestimates turbulent fluctuations in highly turbulent regions. He did not attempt to measure fluctuations in known recirculation regions. However, in the present case, the recirculation zone has not been identified. Nonetheless, it can be asserted that the SAS is in URANS mode.

\section{Conclusion}

The capability of different turbulence models was investigated for jet in crossflow mixing at low velocity ratios. The results are evaluated with respect to the application in fuel premix ducts of combustion systems. In these systems the critical phenomena are potential autoignition in the lean shear layer and subsequent flame stabilization in the recirculation zone.

LES captures the mixing field in all regions very well and is in good agreement with the experimental reference data. The RANS approach underpredicts the turbulent mixing in the three-dimensional shear layer vortices and in the recirculation zone. Especially in the $R=2$ case, the LES results indicate a negative velocity at $x / D=2$ close to the wall. The accurate prediction of this region is very important to capture flame propagation and stabilization mechanisms in the recirculation zone. However the RANS model, however, predicts a positive streamwise velocity. This result corresponds to the findings by Ivanova et. al. ${ }^{23}$ The authors have shown that URANS modeling fails to reproduce flame stabilization in such a 

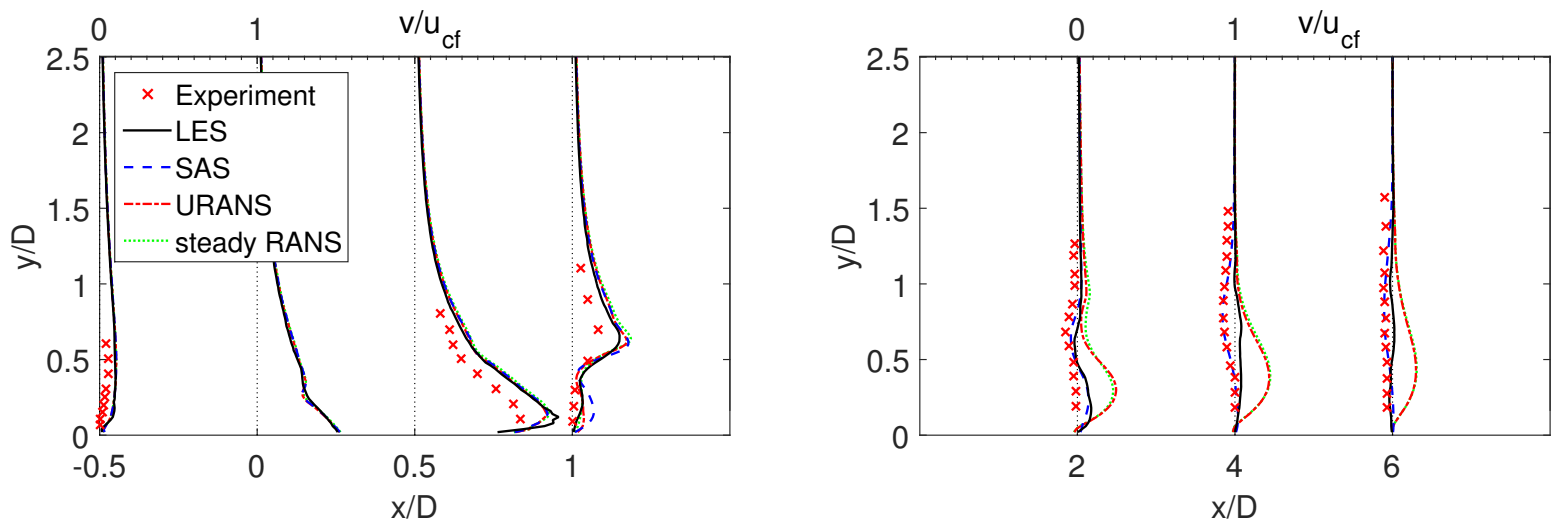

Figure 14. $R=0.5$ : Profiles of time-averaged dimensionless streamwise velocity $\bar{v} / u_{c} f$ at plane of symmetry $z / D=0$ and different streamwise locations $x / D=-0.5$ to 1 (left) and $x / D=2$ to 6 (right).
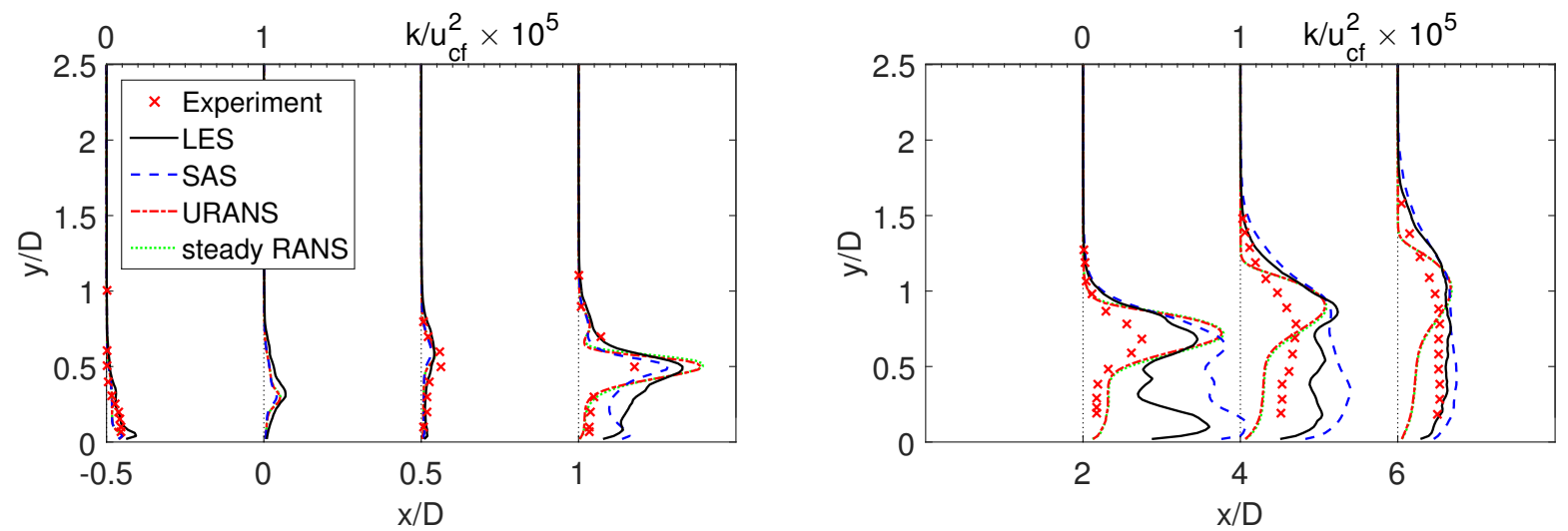

Figure 15. $R=0.5$ : Profiles of time-averaged dimensionless turbulent kinetic energy $k / u_{c f}^{2}$ at plane of symmetry $z / D=0$ and different streamwise locations $x / D=-0.5$ to 1 (left) and $x / D=2$ to 6 (right).

configuration. Furthermore there is almost no difference between unsteady and steady time discretization of the RANS equations.

The accuracy of the hybrid SAS model is similar to RANS modeling in the front shear layer. This is due to a delayed transition into the scale-resolving mode. In the wake it slowly switches into scale resolving mode between $x / D=2-4$. Downstream at about $x / D=6$, good agreement with LES and experimental data is achieved. In the recirculation region the SAS model stays in RANS mode for the smaller velocity ratio, where this zone is less pronounced. For the $R=2$ case the SAS model is almost immediately in LES mode and is therefore a good alternative for the prediction of flame stabilization.

\section{Acknowledgments}

The authors wish to thank ALSTOM Switzerland for their financial support of the project.

\section{References}

\footnotetext{
${ }^{1}$ Prause, J., Noll, B., Aigner, M., and Syed, K., "Sensitivity Analysis of Auto-Ignition Simulation at Gas Turbine Operating Conditions," Journal of Engineering for Gas Turbines and Power, Vol. 137, 2015.

${ }^{2}$ Joos, F., Brunner, P., Schulte-Werning, B., Syed, K., and Ergolu, A., "Development of the Sequential Combustion System for the ABB GT24/GT26 Gas Turbine Family," Volume 4: Heat Transfer, Electric Power, Industrial and Cogeneration, No. Paper No. 96-GT-315, ASME, 1996.

${ }^{3}$ Fleck, J., Griebel, P., Steinberg, A., Stohr, M., Aigner, M., and Ciani, A., "Autoignition Limits of Hydrogen at Relevant Reheat Combustor Operating Conditions," J. Eng. Gas Turbines Power, Vol. 134, No. 4, 2012, pp. 041502.

${ }^{4}$ Andreopoulos, "Heat transfer measuremnets in a heated jet-pipe flow issuing into a cold cross stream," Phys. Fluids, Vol. 26, No. 11, 1983, pp. 3201-3210.
} 
${ }^{5}$ Andreopoulos, J. and Rodi, W., "Experimental investigation of jets in a crossflow," Journal of Fluid Mechanics, Vol. 138, 1984, pp. 93-127.

${ }^{6}$ J.U. Schlüter and T. Schönfeld, "LES of Jets in Cross Flow and Its Application to a Gas Turbine Burner," Flow, Turbulence and Combustion, 2000.

${ }^{7}$ Wegner, B., Huai, Y., and Sadiki, A., "Comparative study of turbulent mixing in jet in cross-flow configurations using LES," International Journal of Heat and Fluid Flow, Vol. 25, No. 5, oct 2004, pp. 767-775.

${ }^{8}$ Olbricht, C., Hahn, F., Sadiki, A., and Janicka, J., "Analysis of subgrid scale mixing using a hybrid LES-Monte-Carlo PDF method," International Journal of Heat and Fluid Flow, Vol. 28, No. 6, dec 2007, pp. 1215-1226.

${ }^{9}$ Fröhlich, J. and von Terzi, D., "Hybrid LES/RANS methods for the simulation of turbulent flows," Progress in Aerospace Sciences, Vol. 44, No. 5, jul 2008, pp. 349-377.

${ }^{10}$ Menter, F. R. and Egorov, Y., "A Scale-Adaptive Simulation Model using Two-Equation Models," 43rd AIAA Aerospace Sciences Meeting and Exhibit, No. 2005-1095, 2005.

${ }^{11}$ Ivanova, E., Domenico, M. D., Noll, B., and Aigner, M., "Unsteady Simulations of Flow Field and Scalar Mixing in Transverse Jets," Volume 2: Combustion, Fuels and Emissions, ASME, 2009.

${ }^{12}$ Duda, B. M., Menter, F. R., Hansen, T., and Esteve, M.-J., "Scale-adaptive simulation of a hot jet in cross flow," J. Phys.: Conf. Ser., Vol. 318, No. 4, dec 2011, pp. 042050.

${ }^{13}$ Duda, B. M., Menter, F. R., Deck, S., Bézard, H., Hansen, T., and Estève, M.-J., "Application of the Scale-Adaptive Simulation to a Hot Jet in Cross Flow," AIAA Journal, Vol. 51, No. 3, mar 2013, pp. 674-685.

${ }^{14}$ Egorov, Y., Menter, F. R., Lechner, R., and Cokljat, D., "The Scale-Adaptive Simulation Method for Unsteady Turbulent Flow Predictions. Part 2: Application to Complex Flows," Flow, Turbulence and Combustion, Vol. 85, No. 1, jun 2010, pp. 139165.

${ }^{15}$ Rusch, D. B., Turbulence model validation for fire simulation by CFD and experimental investigation of a hot jet in crossflow, Ph.D. thesis, Eidgenössische Technische Hochschule ETH Zürich, 2006.

${ }^{16}$ Menter, F. R., "Two-equation eddy-viscosity turbulence models for engineering applications," AIAA Journal, Vol. 32, No. 8, 1994, pp. 1598-1605.

${ }^{17}$ Wilcox, D. C., Turbulence Modeling for CFD, DCW Industries, Inc., 1998.

${ }^{18}$ Jones, W. and Launder, B., "The prediction of laminarization with a two-equation model of turbulence," Int. J. Heat Mass Transfer, Vol. 15, 1972, pp. 301-314.

${ }^{19}$ Menter, F., Kuntz, M., and Langtry, R., "Ten Years of Industrial Experience with the SST Turbulence Model," Turbulence, Heat and Mass Transfer 4, Bergell House, Inc., k. hanjalic, y. nagano, m. tummers ed., 2003.

${ }^{20}$ F.Ducros, Nicoud, F., and T.Poinsot, "Wall-adapting local eddy-viscosity models for simulations in complex geometries," In 6th ICFD Conference on numerical methods for fluid dynamics, 1998, pp. 293-299.

${ }^{21}$ Hunt, J., Wray, A., and Moin, P., "Eddies, stream, and convergence zones in turbulent flows," Tech. rep., Center for Turbulence Research, 1988.

${ }^{22}$ Fric, T. F. and Roshko, A., "Vortical structure in the wake of a transverse jet," Journal of Fluid Mechanics, Vol. 279, 1994, pp. 1-47.

${ }^{23}$ Ivanova, E., Noll, B., Griebel, P., Aigner, M., and Syed, K., "Numerical Simulations of Turbulent Mixing and Autoignition of Hydrogen Fuel at Reheat Combustor Operating Conditions," Volume 2: Combustion, Fuels and Emissions, Parts A and B, ASME, 2011. 